\title{
A weighted Khintchine inequality
}

\author{
Sergey V. Astashkin and Guillermo P. Curbera
}

Abstract. We prove a weighted version of the well-known Khintchine inequality for rearrangement invariant norms.

\section{Introduction and main results}

The classical Khintchine inequality states, for $0<p<\infty$, that there exist constants $A_{p}, B_{p}>0$ such that

$$
A_{p}\left(\sum_{i=1}^{\infty} a_{i}^{2}\right)^{1 / 2} \leq\left(\int_{0}^{1}\left|\sum_{i=1}^{\infty} a_{i} r_{i}(t)\right|^{p} d t\right)^{1 / p} \leq B_{p}\left(\sum_{i=1}^{\infty} a_{i}^{2}\right)^{1 / 2},
$$

for every $\left(a_{i}\right) \in \ell^{2}$, where $\left(r_{i}\right)$ are the Rademacher functions, that is, $r_{i}(t):=$ $\operatorname{sign} \sin \left(2^{i} \pi t\right), t \in[0,1], i \in \mathbb{N}$.

A weighted version of the above inequality was recently proved in [18]. Namely, let $w$ be a weight satisfying the following conditions:

$(\alpha)$ for some $q>p$ we have $w \in L^{q}([0,1])$;

$(\beta)$ the support of $w$ satisfies $m(\operatorname{supp}(w))>2 / 3$.

Then there exist constants $C_{1}, C_{2}>0$, depending on $p$ and $w$, such that for every $a=\left(a_{i}\right) \in \ell^{2}$,

$$
C_{1}^{-1}\|a\|_{2} \leq\left(\int_{0}^{1}\left|\sum_{i=1}^{\infty} a_{i} r_{i}(t)\right|^{p}|w(t)|^{p} d t\right)^{1 / p} \leq C_{2}\|a\|_{2},
$$

where $\|a\|_{2}:=\left(\sum_{i=1}^{\infty} a_{i}^{2}\right)^{1 / 2}$.

In this paper we will consider the extension of the above inequality in two directions. On the one hand, instead of the family of $L^{p}$-spaces we consider the inequality for the essentially larger family of rearrangement invariant spaces. On the other hand, we investigate the restriction on the measure of the support of the 
weight. Note that some restriction on the support of the weight is needed in order to have the lower estimate in (1.1) because there are Rademacher series with large zero sets; see Proposition 7. In this regard the following result, due to Stechkin and Ul'yanov, [16], on sets of uniqueness for Rademacher series is noteworthy: if $g=\sum_{i=1}^{\infty} a_{i} r_{i}$ and $m(\operatorname{supp}(g))<1 / 2$ then $a_{i}=0$ for all $i \geq 1$. The constant $1 / 2$ is sharp, since for $g=r_{1}+r_{2}$ we have $m(\operatorname{supp}(g))=1 / 2$. We replace the condition on the size of the support of $w$ by a condition that depends on the structure of the support. A lower estimate in the weighted Khintchine inequality for weights having support with arbitrarily small measure is then possible.

Consider the following class of subsets of $[0,1]$. By $\Delta_{k}^{n}$ we will denote the dyadic intervals of order $n$, that is, $\Delta_{k}^{n}=\left[(k-1) / 2^{n}, k / 2^{n}\right]$, for $n \in \mathbb{N}$ and $k=1,2, \ldots, 2^{n}$. We say that a measurable set $E \subset[0,1]$ belongs to the class $\mathcal{E}$ if there exist $n \in \mathbb{N}$, $\varepsilon \in\left(0,2^{-n-2}\right), \delta \in(0,1)$, and $\gamma \in(1 / 2,1)$ such that the following conditions are satisfied:

(i) there is a set $I \subset\left\{1, \ldots, 2^{n}\right\}$, card $I>\gamma 2^{n}$, such that, for every $k \in I$, the set $E \cap \Delta_{n}^{k}$ is symmetric with respect to the midpoint of the interval $\Delta_{n}^{k}$ and $m\left(E \cap \Delta_{n}^{k}\right)>\delta$.

(ii) there exists $k_{0}, 1 \leq k_{0} \leq 2^{n}$, such that $m\left(E \cap \Delta_{k_{0}}^{n}\right)>\varepsilon+3 \cdot 2^{-n-2}$.

We will indicate this situation by writing $E \in \mathcal{E}_{\varepsilon, \delta, \gamma}^{n}$.

Let $\Lambda(X)$ be the Rademacher multiplicator space of a rearrangement invariant (r.i.) space $X$ (for the definitions and discussion related to basic concepts, see below). Moreover, for any $w \in \Lambda(X)$ and $\eta>0$ we write

$$
M_{\eta}(w):=\left\{t \in[0,1]:|w(t)| \geq \eta\|w\|_{\Lambda(X)}\right\} .
$$

The main result of this paper is the following weighted version of the Khintchine inequality.

Theorem 1. Let $X$ be an r.i. space on $[0,1]$ such that the Rademacher functions generate in $X$ a subspace isomorphic to $\ell^{2}$. Let $w \in \Lambda(X)$ be such that there exists $\eta>0$ satisfying at least one of the following conditions:

(a) $\alpha_{\eta}:=\max \left\{m\left(M_{\eta}(w) \cap\left[0, \frac{1}{2}\right]\right), m\left(M_{\eta}(w) \cap\left[\frac{1}{2}, 1\right]\right)\right\}>\frac{1}{4}$;

(b) the set $M_{\eta}(w)$ contains a set $E \in \mathcal{E}$.

Then, for every $a=\left(a_{i}\right) \in \ell^{2}$,

$$
D_{w}\|w\|_{\Lambda(X)}\|a\|_{2} \leq\left\|w \cdot \sum_{i=1}^{\infty} a_{i} r_{i}\right\|_{X} \leq C_{X}\|w\|_{\Lambda(X)}\|a\|_{2},
$$

where $C_{X}$ depends only on $X$, and, in the case (a), $D_{w}=\frac{\eta}{4}\left(\alpha_{\eta}-1 / 4\right)^{2}$, and, in the case (b), $D_{w}=\frac{\eta}{32} \min \{2 \varepsilon, \delta(\gamma-1 / 2)\} \cdot \min \left\{2^{n-1} \varepsilon, \frac{1}{4}(\gamma-1 / 2)\right\}$, where $E \in \mathcal{E}_{\varepsilon, \delta, \gamma}^{n}$, with $n \in \mathbb{N}, \varepsilon \in\left(0,2^{-n-2}\right), \delta \in(0,1)$, and $\gamma \in(1 / 2,1)$. 
Corollary 2. Let $X$ be an r.i. space on $[0,1]$ such that the Rademacher functions generate in $X$ a subspace isomorphic to $\ell^{2}$. Let $w \in \Lambda(X)$ be such that

$$
\alpha_{w}:=\max \left\{m\left(\operatorname{supp}(w) \cap\left[0, \frac{1}{2}\right]\right), m\left(\operatorname{supp}(w) \cap\left[\frac{1}{2}, 1\right]\right)\right\}>\frac{1}{4} .
$$

Then, for all sufficiently small $\eta>0$, inequality (1.2) holds with $D_{w}=\frac{\eta}{4}\left(\alpha_{w}-\frac{1}{4}\right)^{2}$.

Regarding the concepts appearing in Theorem 1 and Corollary 2, recall that the distribution function of a measurable function $f$ is $m_{f}(\tau):=m(\{t \in[0,1]$ : $|f(t)|>\tau\}$ ), where $m$ is the Lebesgue measure on $[0,1]$. A rearrangement invariant (r.i.) space $X$ is a Banach space of classes of measurable functions on $[0,1]$ such that if $m_{g}(\tau) \leq m_{f}(\tau)$, for all $\tau>0$, and $f \in X$ then $g \in X$ and $\|g\|_{X} \leq\|f\|_{X}$. For normalization purposes we will assume that $\left\|\chi_{[0,1]}\right\|_{X}=1$. The class of r.i. spaces contains many well-known families of function spaces: $L^{p}$, Orlicz, $L^{p, q}$, Lorentz, Marcinkiewicz, Zygmund, and many others. For r.i. spaces, see [6] and [12].

Let $\mathcal{R}$ denote the set of all functions of the form $\sum_{i=1}^{\infty} a_{i} r_{i}$, where the series converges a.e., that is, $\left(a_{i}\right) \in \ell^{2}$, see Theorem V.8.2 in [19]. For an r.i. space $X$, let $\mathcal{R}(X)$ be the closed linear subspace of $X$ given by $\mathcal{R} \cap X$. The Khintchine inequality shows, for $X=L^{p}, 1 \leq p<\infty$, that $\mathcal{R}(X)$ is isomorphic to $\ell^{2}$. If $X=L^{\infty}$, then $\mathcal{R}(X)=\ell^{1}$. A result of Rodin and Semenov characterizes when $\mathcal{R}(X) \approx \ell^{2}$. Let $L_{N}$ be the Orlicz space associated to the function $N(t):=$ $\exp \left(t^{2}\right)-1$, and let $\left(L_{N}\right)_{0}$ be the closure of $L^{\infty}$ in $L_{N}$. Then $\mathcal{R}(X) \approx \ell^{2}$, that is,

$$
c_{X}\left\|\left(a_{i}\right)\right\|_{2} \leq\left\|\sum_{i=1}^{\infty} a_{i} r_{i}\right\|_{X} \leq C_{X}\left\|\left(a_{i}\right)\right\|_{2},
$$

for some constants $C_{X}, c_{X}>0$, if and only if $\left(L_{N}\right)_{0} \subset X,[13]$.

If $X$ is a r.i. space on $[0,1]$, the Rademacher multiplicator space $\Lambda(X)$ consists of all measurable functions $f:[0,1] \rightarrow \mathbb{R}$ such that $f \sum_{i=1}^{\infty} a_{i} r_{i} \in X$, for every $\sum_{i=1}^{\infty} a_{i} r_{i} \in \mathcal{R}(X)$. It is a Banach function space on $[0,1]$ when endowed with the norm

$$
\|f\|_{\Lambda(X)}:=\sup \left\{\left\|f \sum_{i=1}^{\infty} a_{i} r_{i}\right\|_{X}: \sum_{i=1}^{\infty} a_{i} r_{i} \in X,\left\|\sum_{i=1}^{\infty} a_{i} r_{i}\right\|_{X} \leq 1\right\} .
$$

Remark 3. (a) The space $\Lambda(X)$ need not be r.i. However, in many cases it is possible to identify the largest r.i. space embedded in $\Lambda(X)$ (its symmetric kernel) denoted by $\operatorname{Sym}(X)$. For example, $\operatorname{Sym}\left(L^{p}\right)$ is the Zygmund space $L^{p}(\log L)^{1 / 2}$, $1 \leq p<\infty$. For more facts on $\Lambda(X)$ and $\operatorname{Sym}(X)$, see [1]-[5], [10], and [11].

(b) For the case $X=L^{p}, 1 \leq p<\infty$, the condition $w \in \Lambda\left(L^{p}\right)$ in Theorem 1 is much weaker than the condition in the above cited result of Veraar, [18]: $w \in L_{q}$ for some $q>p$. To see this take into account that $L^{q} \subseteq L^{p}(\log L)^{1 / 2} \subseteq \Lambda\left(L^{p}\right)$.

(c) Note that all sets satisfying the condition $m(\operatorname{supp}(w))>2 / 3$, which is used in [18], and even the weaker one $m(\operatorname{supp}(w))>1 / 2$, satisfy also condition (a) of Theorem 1. Condition (b) in Theorem 1 on a weight $w$ depends not so much on the size of the support of $w$ as on its structure, showing that the lower estimate 
in the weighted Khintchine inequality can hold for weights having support with arbitrarily small measure. It is instructive to emphasize that either of the conditions (a) and (b) guarantees that for every Rademacher zero set $F$ (this means that there is a Rademacher sum $g=\sum_{i=1}^{\infty} a_{i} r_{i}$ vanishing on $\left.F\right)$ we have $\operatorname{supp}(g) \backslash F \neq \emptyset$ (regarding Rademacher zero sets see also the appendix).

\section{Proofs}

The proof of the upper bound in Theorem 1 follows directly from the definition of the Rademacher multiplicator space. Indeed, since by hypothesis we have (1.3), from (1.4) it follows that

$$
\sup _{a \in \ell^{2}, a \neq 0} \frac{\left\|w \cdot \sum_{i=1}^{\infty} a_{i} r_{i}\right\|_{X}}{\left(\sum_{i=1}^{\infty} a_{i}^{2}\right)^{1 / 2}} \leq C_{X} \cdot \sup _{a \in \ell^{2}, a \neq 0} \frac{\left\|w \cdot \sum_{i=1}^{\infty} a_{i} r_{i}\right\|_{X}}{\left\|\sum_{i=1}^{\infty} a_{i} r_{i}\right\|_{X}}=C_{X}\|w\|_{\Lambda(X)} .
$$

Note that the definition of the Rademacher multiplicator space $\Lambda(X)$ also shows that this upper bound is (up to a constant) optimal.

To prove the lower bound we will need the following assertion.

Proposition 4. Let $E \subset[0,1]$ be a measurable set.

(a) Suppose that E satisfies

$$
\alpha:=\max \left\{m\left(E \cap\left[0, \frac{1}{2}\right]\right), m\left(E \cap\left[\frac{1}{2}, 1\right]\right)\right\}>\frac{1}{4} .
$$

Then for $0<B \leq \frac{1}{2}(\alpha-1 / 4)$ and for every $a=\left(a_{i}\right) \in \ell^{2}$ we have

$$
m\left(\left\{t \in E:\left|\sum_{i=1}^{\infty} a_{i} r_{i}(t)\right| \geq B\|a\|_{2}\right\}\right)>\frac{1}{2}\left(\alpha-\frac{1}{4}\right) .
$$

(b) Suppose that $E \in \mathcal{E}$. Then, for every $a=\left(a_{i}\right) \in \ell^{2}$, we have

$$
m\left(\left\{t \in E:\left|\sum_{i=1}^{\infty} a_{i} r_{i}(t)\right| \geq B\|a\|_{2}\right\}\right)>\frac{1}{4} \min \left\{2 \varepsilon, \delta\left(\gamma-\frac{1}{2}\right)\right\},
$$

for $0<B \leq B_{n, \varepsilon, \gamma}:=\min \left(2^{n-1} \epsilon, \frac{1}{4}(\gamma-1 / 2)\right)$, where $E \in \mathcal{E}_{\varepsilon, \delta, \gamma}^{n}$, for $n \in \mathbb{N}$, $\varepsilon \in\left(0,2^{-n-2}\right), \delta \in(0,1)$, and $\gamma \in(1 / 2,1)$.

Proof. We will apply two well-known results. First, the Paley-Zygmund inequality (see, for instance, Lemma V.8.26 in [19] or Lemma 1 in [7]) will be used in the following form: for any $A>B \geq 0$ and for arbitrary nonnegative random variable $f$ with $\mathbb{E} f \geq A$ and $\mathbb{E} f^{2}=1$ we have

$$
m(f \geq B) \geq(A-B)^{2} .
$$


Moreover, by [17], the best constant $A_{1}$ in the Khintchine inequality for $p=1$ is equal to $1 / \sqrt{2}$. Therefore, for every $a=\left(a_{i}\right) \in \ell^{2}$

$$
\int_{0}^{1}\left|\sum_{i=1}^{\infty} a_{i} r_{i}(t)\right| d t \geq \frac{1}{\sqrt{2}}\|a\|_{2},
$$

and we can apply Paley-Zygmund's inequality to the function $f:=\left|\sum_{i=1}^{\infty} a_{i} r_{i}\right| /\|a\|_{2}$ with $A=1 / \sqrt{2}$. Then, for any $B$ with $0 \leq B<1 / \sqrt{2}$, we have

$$
m\left(\left\{t \in[0,1]:\left|\sum_{i=1}^{\infty} a_{i} r_{i}(t)\right| \geq B\|a\|_{2}\right\}\right) \geq\left(\frac{1}{\sqrt{2}}-B\right)^{2} .
$$

We first prove (a). Assume, for example, that $\alpha=m(E \cap[0,1 / 2])$. Define $Q_{B}:=\left\{t \in[0,1 / 2]:\left|\sum_{i=1}^{\infty} a_{i} r_{i}(t)\right| \geq B\|a\|_{2}\right\}$. Due to elementary properties of the Rademacher functions, the set $\left\{t \in[0,1]:\left|\sum_{i=1}^{\infty} a_{i} r_{i}(t)\right| \geq B\|a\|_{2}\right\}$ is symmetric with respect to the point $1 / 2$ and thus from (2.1) it follows that

$$
m\left(Q_{B}\right) \geq \frac{1}{2}\left(\frac{1}{\sqrt{2}}-B\right)^{2} .
$$

Hence, provided that $0<B \leq \frac{1}{2}(\alpha-1 / 4)$ we have

$m\left(E \cap Q_{B}\right) \geq m(E \cap[0,1 / 2])+m\left(Q_{B}\right)-\frac{1}{2} \geq \alpha+\frac{1}{2}\left(\frac{1}{\sqrt{2}}-B\right)^{2}-\frac{1}{2}>\frac{1}{2}\left(\alpha-\frac{1}{4}\right)$,

and the inequality in case (a) is proved. Similar estimates establish the result when $\alpha=m(E \cap[1 / 2,1])$.

Now, we proceed with the proof of (b). Assume that $E \in \mathcal{E}_{\varepsilon, \delta, \gamma}^{n}$, for some $n \in \mathbb{N}, \varepsilon>0, \delta \in(0,1)$, and $\gamma \in(1 / 2,1)$. Let $g=\sum_{i=1}^{\infty} a_{i} r_{i}$ be an arbitrary Rademacher series, where $a=\left(a_{i}\right) \in \ell^{2}$, and let $Q_{E, B}:=\left\{t \in E:|g(t)| \geq B\|a\|_{2}\right\}$.

First, we suppose that

$$
\sum_{i=1}^{n} a_{i}^{2} \leq \sum_{i=n+1}^{\infty} a_{i}^{2}
$$

By hypothesis, there exists $k_{0}, 1 \leq k_{0} \leq 2^{n}$, such that $m\left(E \cap \Delta_{k_{0}}^{n}\right)>\varepsilon+3$. $2^{-n-2}$. Since the function $\sum_{i=1}^{n} a_{i} r_{i}$ is constant on the interval $\Delta_{k_{0}}^{n}$, say $a_{0}$, then, using (2.2), we obtain that

$$
\begin{aligned}
m\left(Q_{E, B}\right) & \geq m\left\{t \in E:|g(t)| \geq \sqrt{2} B\left(\sum_{i=n+1}^{\infty} a_{i}^{2}\right)^{1 / 2}\right\} \\
& \geq m\left\{t \in E \cap \Delta_{k_{0}}^{n}:\left|a_{0}+\sum_{i=n+1}^{\infty} a_{i} r_{i}(t)\right| \geq \sqrt{2} B\left(\sum_{i=n+1}^{\infty} a_{i}^{2}\right)^{1 / 2}\right\} .
\end{aligned}
$$

Therefore, we can choose a set $F \subset[0,1]$ with $m(F)=2^{n} m\left(E \cap \Delta_{k_{0}}^{n}\right)>3 / 4$ and such that

$$
m\left(Q_{E, B}\right) \geq 2^{-n} m\left(\left\{t \in F:\left|a_{0}+\sum_{i=n+1}^{\infty} a_{i} r_{i}(t)\right| \geq \sqrt{2} B\left(\sum_{i=n+1}^{\infty} a_{i}^{2}\right)^{1 / 2}\right\}\right)
$$


At the same time, from (2.1) it follows that

$$
m\left(\left\{t \in[0,1]:\left|\sum_{i=n+1}^{\infty} a_{i} r_{i}(t)\right| \geq \sqrt{2} B\left(\sum_{i=n+1}^{\infty} a_{i}^{2}\right)^{1 / 2}\right\}\right) \geq\left(\frac{1}{\sqrt{2}}-\sqrt{2} B\right)^{2} .
$$

Combining this inequality with elementary symmetry properties of the Rademacher functions, we obtain that

$$
m\left(\left\{t \in[0,1]:\left|a_{0}+\sum_{i=n+1}^{\infty} a_{i} r_{i}(t)\right| \geq \sqrt{2} B\left(\sum_{i=n+1}^{\infty} a_{i}^{2}\right)^{1 / 2}\right\}\right) \geq \frac{1}{2}\left(\frac{1}{\sqrt{2}}-\sqrt{2} B\right)^{2} .
$$

Therefore, arguing in the same way as in the proof of (a), we obtain that

$$
\begin{aligned}
m\left(\left\{t \in F: \mid a_{0}\right.\right. & \left.\left.+\sum_{i=n+1}^{\infty} a_{i} r_{i}(t) \mid \geq \sqrt{2} B\left(\sum_{i=n+1}^{\infty} a_{i}^{2}\right)^{1 / 2}\right\}\right) \\
& \geq m(F)+\frac{1}{2}\left(\frac{1}{\sqrt{2}}-\sqrt{2} B\right)^{2}-1 \geq m(F)-\frac{3}{4}-B .
\end{aligned}
$$

Since

$$
m(F)-\frac{3}{4}=2^{n}\left(m\left(E \cap \Delta_{k_{0}}^{n}\right)-3 \cdot 2^{-n-2}\right)>2^{n} \varepsilon,
$$

and, by hypotheses $B<2^{n-1} \varepsilon$, from the previous inequality it follows that

$$
m\left(\left\{t \in F:\left|a_{0}+\sum_{i=n+1}^{\infty} a_{i} r_{i}(t)\right| \geq \sqrt{2} B\left(\sum_{i=n+1}^{\infty} a_{i}^{2}\right)^{1 / 2}\right\}\right) \geq 2^{n-1} \varepsilon .
$$

Hence, using (2.3), we obtain, provided $0<B<2^{n-1} \varepsilon$, that

$$
m\left(Q_{E, B}\right) \geq \frac{\varepsilon}{2} .
$$

Consider now the case when inequality (2.2) does not hold. Then

$$
m\left(Q_{E, B}\right) \geq m\left\{t \in E:|g(t)| \geq \sqrt{2} B\left(\sum_{i=1}^{n} a_{i}^{2}\right)^{1 / 2}\right\} .
$$

By hypothesis, if $I:=\left\{k=1,2, \ldots, 2^{n}: m\left(E \cap \Delta_{k}^{n}\right)>\delta\right\}$, then

$$
\text { card } I>\gamma 2^{n} \text {. }
$$

Again, by (2.1), for some $I^{\prime} \subseteq\left\{1,2, \ldots, 2^{n}\right\}$, we have that

$$
\begin{aligned}
m(\{t \in[0,1] & \left.\left.:\left|\sum_{i=1}^{n} a_{i} r_{i}(t)\right| \geq \sqrt{2} B\left(\sum_{i=1}^{n} a_{i}^{2}\right)^{1 / 2}\right\}\right) \\
& =\sum_{i \in I^{\prime}} m\left(\Delta_{i}^{n}\right)=\operatorname{card} I^{\prime} \cdot 2^{-n} \geq\left(\frac{1}{\sqrt{2}}-\sqrt{2} B\right)^{2},
\end{aligned}
$$


whence

$$
\operatorname{card} I^{\prime} \geq 2^{n}\left(\frac{1}{\sqrt{2}}-\sqrt{2} B\right)^{2}
$$

It is easy to check that the condition $0<B \leq \frac{1}{4}\left(\gamma-\frac{1}{2}\right)$ guarantees that

$$
\left(\frac{1}{\sqrt{2}}-\sqrt{2} B\right)^{2}+\gamma-1>\frac{1}{2}\left(\gamma-\frac{1}{2}\right)
$$

Therefore, by (2.6),

$$
\begin{aligned}
\operatorname{card}\left(I^{\prime} \cap I\right) \geq \operatorname{card}\left(I^{\prime}\right)+\operatorname{card}(I)-2^{n} & >2^{n}\left(\left(\frac{1}{\sqrt{2}}-\sqrt{2} B\right)^{2}+\gamma-1\right) \\
& >2^{n-1}\left(\gamma-\frac{1}{2}\right) .
\end{aligned}
$$

By hypothesis, for every $k \in I$, the set $E \cap \Delta_{k}^{n}$ is symmetric with respect to the midpoint of the interval $\Delta_{k}^{n}$. Therefore, if $t \in E \cap \Delta_{k}^{n}$, where $k \in I \cap I^{\prime}$ is fixed, then the symmetric point $t^{\prime}$ also belongs to the set $E \cap \Delta_{k}^{n}$. Note that the sum $\sum_{i=1}^{n} a_{i} r_{i}$ is constant on the interval $E \cap \Delta_{k}^{n}$, having, say, value $b$, and $|b| \geq \sqrt{2} B\left(\sum_{i=1}^{n} a_{i}^{2}\right)^{1 / 2}$. On the other hand, since $r_{i}\left(t^{\prime}\right)=-r_{i}(t)$, if $i>n$, we have that $g(t)=b+c$ and $g\left(t^{\prime}\right)=b-c$ for some constant $c$. Obviously, then at least one of the inequalities

$$
|g(t)| \geq \sqrt{2} B\left(\sum_{i=1}^{n} a_{i}^{2}\right)^{1 / 2}, \quad\left|g\left(t^{\prime}\right)\right| \geq \sqrt{2} B\left(\sum_{i=1}^{n} a_{i}^{2}\right)^{1 / 2}
$$

holds. Thus, if $0<B \leq \frac{1}{4}(\gamma-1 / 2)$, by $(2.5)$ and (2.7), we obtain that

$$
\begin{aligned}
m\left(Q_{E, B}\right) & \geq \sum_{k \in I \cap I^{\prime}} m\left\{t \in E \cap \Delta_{k}^{n}:|g(t)| \geq \sqrt{2} B\left(\sum_{i=1}^{n} a_{i}^{2}\right)^{1 / 2}\right\} \\
& \geq \frac{1}{2} \sum_{k \in I \cap I^{\prime}} m\left\{t \in E \cap \Delta_{k}^{n}:\left|\sum_{i=1}^{n} a_{i} r_{i}(t)\right| \geq \sqrt{2} B\left(\sum_{i=1}^{n} a_{i}^{2}\right)^{1 / 2}\right\} \\
& \geq \delta \cdot 2^{-n-1} \operatorname{card}\left(I \cap I^{\prime}\right)>\frac{\delta}{4}\left(\gamma-\frac{1}{2}\right) .
\end{aligned}
$$

Combining the last estimate with the inequality (2.4), we obtain the result.

Corollary 5. Let $E \subseteq[0,1]$ be a measurable set such that at least one of the following conditions holds:

(a) $\alpha:=\max \left\{m\left(E \cap\left[0, \frac{1}{2}\right]\right), m\left(E \cap\left[\frac{1}{2}, 1\right]\right)\right\}>\frac{1}{4}$;

(b) $E \in \mathcal{E}$.

Then, for every $a=\left(a_{i}\right) \in \ell^{2}$, we have that

$$
\left\|\sum_{i=1}^{\infty} a_{i} r_{i} \chi_{E}\right\|_{1} \geq d_{E}\|a\|_{2}
$$


where, in the case (a), we have $d_{E}=\frac{1}{4}(\alpha-1 / 4)^{2}$, and, in the case (b), we have $d_{E}=\frac{1}{32} \min \{2 \varepsilon, \delta(\gamma-1 / 2)\} B_{n, \varepsilon, \gamma}$, where $E \in \mathcal{E}_{\varepsilon, \delta, \gamma}^{n}$, for $n \in \mathbb{N}, \varepsilon \in\left(0,2^{-n-2}\right)$, $\delta \in(0,1)$, and $\gamma \in(1 / 2,1)$.

Remark 6. Proposition 4 should be compared with the following inequality from the work of Burkholder (Theorem 1 in [7]): there exist universal constants $\xi_{1}>0$ and $\xi_{2}>0$ such that for every set $E \subset[0,1]$ with $m(E)>0$ there exists $N:=N(E)$ such that

$$
m\left(\left\{t \in E:\left|\sum_{i=N}^{\infty} a_{i} r_{i}(t)\right| \geq \xi_{2}\left(\sum_{i=N}^{\infty} a_{i}^{2}\right)^{1 / 2}\right\}\right) \geq \xi_{1} \cdot m(E),
$$

for any $\left(a_{i}\right) \in \ell^{2}$. Of course, because of existing Rademacher zero sets (see Remark 3(c)), the latter inequality does not hold with $N=1$ for all measurable $E \subset[0,1]$. Nevertheless, Proposition 4 shows that there are some sets (including sets with arbitrarily small measure) for which an analogous estimate is obtained for the Rademacher series starting at $N=1$. For issues regarding the local version of the Khintchine inequality, see [5], [9], [14], and [15].

Now, we prove the lower estimate in Theorem 1, for example, in the case when the condition (a) is satisfied. Let $\eta>0$ be such that

$$
\alpha_{\eta}:=\max \left\{m\left(M_{\eta}(w) \cap\left[0, \frac{1}{2}\right]\right), m\left(M_{\eta}(w) \cap\left[\frac{1}{2}, 1\right]\right)\right\}>\frac{1}{4} .
$$

Since for an r.i. space $X$ on $[0,1]$ with $\left\|\chi_{[0,1]}\right\|_{X}=1$ we have $\|x\|_{1} \leq\|x\|_{X}(x \in X)$, from Corollary 5 we deduce that

$$
\begin{aligned}
\left\|w \cdot \sum_{i=1}^{\infty} a_{i} r_{i}\right\|_{X} & \geq\left\|w \chi_{M_{\eta}(w)} \cdot \sum_{i=1}^{\infty} a_{i} r_{i}\right\|_{X} \\
& \geq \eta\|w\|_{\Lambda(X)}\left\|\chi_{M_{\eta}(w)} \cdot \sum_{i=1}^{\infty} a_{i} r_{i}\right\|_{1} \geq \frac{\eta}{4}\left(\alpha_{\eta}-\frac{1}{4}\right)^{2}\|w\|_{\Lambda(X)}\|a\|_{2},
\end{aligned}
$$

and the proof of the lower estimate in (1.2) is complete. In the case when the condition (b) holds this estimate can be proved by completely analogous arguments.

The proof of Corollary 2 follows from the equality $\operatorname{supp}(w)=\cup_{\eta>0} M_{\eta}(w)$ and the steps of the proof of Proposition 4(a).

\section{Appendix: Rademacher zero sets with measure $1 / 2$}

Proposition 7. Let $g=\sum_{k=1}^{\infty} a_{k} r_{k}$. If

$$
m(\{t \in[0,1]: g(t)=0\})=\frac{1}{2},
$$

then there exist $1 \leq m<n$ and $a \in \mathbb{R}$ such that $g=a\left(r_{n}+r_{m}\right)$ or $g=a\left(r_{n}-r_{m}\right)$. 
Proof. Since infinite Rademacher series are almost everywhere non-null (by Theorem 4 in [16], see also Corollary 1 in [7], and Corollary in [8]), there is an $n \in \mathbb{N}$ such that $g=\sum_{k=1}^{n} a_{k} r_{k}$. Let $b_{k}:=-a_{k} / a_{n}$ if $1 \leq k<n$. By considering $h=r_{n}-\sum_{k=1}^{m} b_{k} r_{k}$, where $b_{m} \neq 0$ and $1 \leq m<n$, from our hypothesis it follows that there exists a set $E \subset[0,1]$ with $m(E)=1 / 2$ such that

$$
r_{n}(t)=\sum_{k=1}^{m} b_{k} r_{k}(t) \quad(t \in E) \text {. }
$$

We have $E=\cup_{i \in I} \Delta_{i}^{n}$ for some set $I \subset\left\{1,2, \ldots, 2^{n}\right\}$. For any $i \in I$ there is $j_{i} \in\left\{1,2, \ldots, 2^{m}\right\}$ such that $\Delta_{i}^{n} \subset \Delta_{j_{i}}^{m}$. Since the sum from the right side of equality (3.1) is constant on the interval $\Delta_{j_{i}}^{m}$, it is equal to +1 or -1 on $\Delta_{j_{i}}^{m}$, depending on the value of $r_{n}$ on the interval $\Delta_{i}^{n}$. Therefore, on half of $\Delta_{j_{i}}^{m}$ we have $r_{n}(t)=\sum_{k=1}^{m} b_{k} r_{k}(t)$ and on the other half $-r_{n}(t)=\sum_{k=1}^{m} b_{k} r_{k}(t)$. Since $m(E)=$ $1 / 2$, it follows that $\left\{j_{i}: i \in I\right\}=\left\{1,2, \ldots, 2^{m}\right\}$. Consequently $\left|\sum_{k=1}^{m} b_{k} r_{k}(t)\right|=1$, for all $t \in[0,1]$, and, by Corollary 3 in [16], we have

$$
m\left(\left\{t \in[0,1]: \sum_{k=1}^{m} b_{k} r_{k}(t)=1\right\}\right)=m\left(\left\{t \in[0,1]: \sum_{k=1}^{m} b_{k} r_{k}(t)=-1\right\}\right)=\frac{1}{2} .
$$

Moreover, assuming that $\sum_{k=1}^{m} b_{k} r_{k}(t)=1$ if $t \in \Delta_{i}^{m}$ for some $1 \leq i<2^{m}$, we see that on the next interval $\Delta_{i+1}^{m}$ only the function $r_{m}(t)$ changes its value. Hence, $\sum_{k=1}^{m} b_{k} r_{k}(t)=-1$ if $t \in \Delta_{i+1}^{m}$. Therefore, the sum $\sum_{k=1}^{m} b_{k} r_{k}(t)$ takes on the intervals $\Delta_{i}^{m}$ alternately the values \pm 1 . Thus, this sum coincides either with $r_{m}$ or with $-r_{m}$, and the proof is complete.

\section{References}

[1] Astashkin, S. V.: On the multiplier space generated by the Rademacher system. Math. Notes 75 (2004), 158-165.

[2] Astashinin, S. V. and Curbera, G. P.: Symmetric kernel of Rademacher multiplicator spaces. J. Funct. Anal. 226 (2005), 173-192.

[3] Astashin, S. V. and Curbera, G. P.: Rademacher multiplicator spaces equal to $L^{\infty}$. Proc. Amer. Math. Soc 136 (2008), 3493-3501.

[4] Astashinin, S. V. and Curbera, G. P.: Rearrangement invariance of Rademacher multiplicator spaces. J. Funct. Anal. 256 (2009), 4071-4094.

[5] Astashkin, S. V. And Curbera, G. P.: Local Khintchine inequality and tail Rademacher multiplicator space in rearrangement invariant spaces. To appear in Ann. Mat. Pura Appl.

[6] Bennett, C. and Sharpley, R.: Interpolation of operators. Pure and Applied Mathematics 129, Academic Press, Boston, 1988.

[7] Burkholder, D. L.: Independent sequences with the Stein property. Ann. Math. Statist. 39 (1968), 1282-1288.

[8] Carefoot, W. C. and Flett, T. M.: A note on Rademacher series. J. London Math. Soc. 42 (1967), 542-544. 
[9] Carrillo-Alanís, J.: On local Khintchine inequalities for spaces of exponential integrability. Proc. Amer. Math. Soc. 139 (2011), 2753-2757.

[10] Curbera, G. P.: A note on function spaces generated by Rademacher series. Proc. Edinburgh. Math. Soc. (2) 40 (1997), 119-126.

[11] Curbera, G.P. And Rodin, V.A.: Multiplication operators on the space of Rademacher series in rearrangement invariant spaces. Math. Proc. Cambridge Phil. Soc. 134 (2003), 153-162.

[12] Krĕ̌n, S. G., Petunin, Ju. I. And Semënov, E. M.: Interpolation of linear operators. Translations of Mathematical Monographs 54, American Mathematical Society, Providence, R. I., 1982.

[13] Rodin, V. A. And Semënov, E. M.: Rademacher series in symmetric spaces. Anal. Math. 1 (1975), 207-222.

[14] Sagher, Y. and Zhou, K.: A local version of a theorem of Khintchine. In Analysis and partial differential equations, 327-330. Lecture Notes in Pure and Appl. Math. 122, Dekker, New York, 1990.

[15] Sagher, Y. And Zhou, K.: Exponential integrability of Rademacher series. In Convergence in ergodic theory and probability (Columbus, OH, 1993), 389-395. Ohio State Univ. Math. Res. Inst. Publ. 5, De Gruyter, Berlin, 1996.

[16] Stechkin, S. B. And Ul'yanov, P. L.: On sets of uniqueness. Izv. Akad. Nauk SSSR Ser. Mat. 26 (1962), 211-222 (in Russian). English translation in Eleven papers in analysis, 203-217. Amer. Math. Soc. Translations Ser. 2, 95, American Mathematical Society, Providence, R. I., 1970.

[17] Szarek, S. J.: On the best constant in the Khintchine inequality. Studia Math. 58 (1976), 197-208.

[18] Veraar, M.: On Khintchine inequalities with a weight. Proc. Amer. Math. Soc. 138 (2011), 4119-4121.

[19] Zygmund, A.: Trigomometric series. Cambrigde University Press, Cambridge, 1977.

Received April 23, 2012.

Sergey V. Astashkin: Department of Mathematics and Mechanics, Samara State University, ul. Akad. Pavlova 1, 443011 Samara, Russia.

E-mail: astash@samsu.ru

Guillermo P. Curbera: Facultad de Matemáticas, Universidad de Sevilla, Aptdo. 1160, Sevilla 41080, Spain.

E-mail: curbera@us.es

Partially supported by MTM2012-36732-C03-03 (Ministerio de Economía y Competitividad), FQM-262, FQM-4643, FQM-7276 (Junta de Andalucía), and Feder Funds (European Union). 